\title{
Erratum: Dissipative Landau-Zener problem and thermally assisted Quantum Annealing [Phys. Rev. B 96, 054301 (2017)]
}

\author{
Luca Arceci, Simone Barbarino, Rosario Fazio, and Giuseppe E. Santoro
}

(Received 4 July 2018; published 16 July 2018)

DOI: 10.1103/PhysRevB.98.019902

Figure 6 has a typographical error in the key: The labels corresponding to RWA and without the RWA are wrongly switched. The caption as well as all the rest of the paper are nevertheless correct and unaffected by this error. We show the exact figure here below with its original caption from the paper.

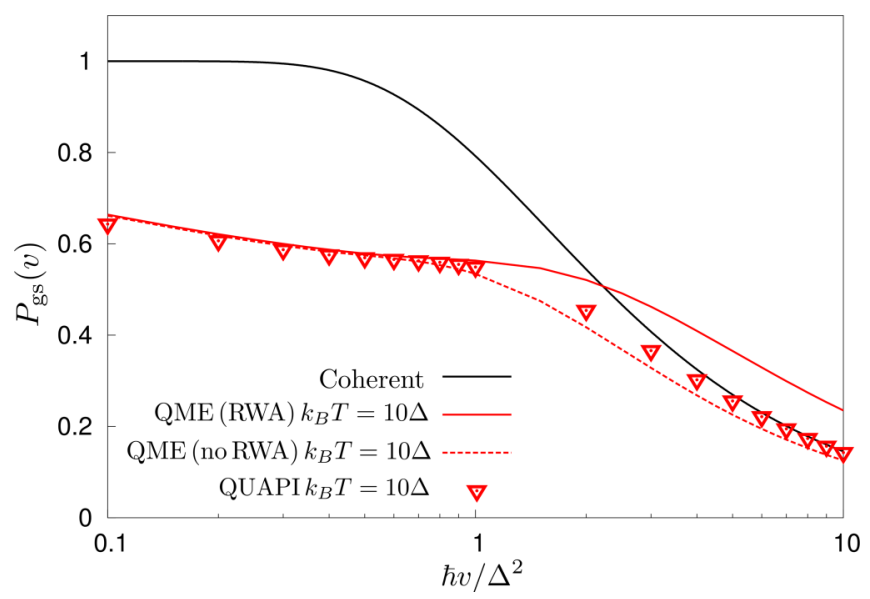

FIG. 6. Comparison of quantum master equation results obtained with and without the rotating-wave approximation (RWA) for a longitudinal bath coupling with $\alpha=0.02$ and $k_{B} T=10 \Delta$. The points denote numerically exact quasiadiabatic path-integral data. The RWA line shows a quite clear overshooting above the coherent evolution result which is an artifact of the approximation. 